\title{
Reporte breve: perspectivas médicas en asma grave
}

\section{Brief report: medical perspectives on severe asthma}

\author{
Erika Meneses-Tamayo, ${ }^{*}$ Willan Chavez-Torres, ${ }^{*}$ Désirée Larenas-Linnemann, ${ }^{\ddagger}$ \\ María del Carmen Cano-Salas, ${ }^{*}$ José Antonio Ortega-Martell, ${ }^{\S}$ Laura Gochicoa-Rangel* \\ *Instituto Nacional de Enfermedades Respiratorias Ismael Cosío Villegas, Ciudad de México, México; \\ ${ }^{\ddagger}$ Centro de Excelencia en Asma y Alergia, Hospital Médica Sur, Ciudad de México, México; \\ ${ }^{\S}$ Instituto de Ciencias de la Salud de la Universidad Autónoma del Estado de Hidalgo.
}

\begin{abstract}
RESUMEN. El asma grave es una patología compleja con características clínicas heterogéneas y diferentes mecanismos subyacentes (endotipos), es representado por un pequeño grupo de pacientes que muestran exacerbaciones frecuentes y obstrucción de las vías respiratorias. Siendo refractaria al tratamiento estándar, requiere dosis altas de corticosteroides inhalados en combinación con un segundo medicamento controlador; existe un subgrupo de pacientes con asma grave no controlada que no presentan mejoría a pesar de este tratamiento. Se han logrado nuevas estrategias para controlar el asma grave al considerar los componentes de la enfermedad y los mecanismos específicos (endotipos) de cada presentación clínica (fenotipo). Una parte importante ha sido el uso de biomarcadores no invasivos como IgE total sérica, el recuento de eosinófilos en sangre y en esputo, y la realización de la determinación de la fracción exhalada de óxido nítrico (FeNO) tanto para el diagnóstico como para el seguimiento. El desarrollo de terapias biológicas dirigidas a vías inflamatorias específicas en el asma ha sido un gran avance en los últimos años, mismas que administradas en pacientes seleccionados reducen las exacerbaciones y mejoran el control de la enfermedad. En este documento se dan a conocer las perspectivas del asma grave visto por un grupo de expertos en el área de la neumología y alergología.
\end{abstract}

Palabras clave: Asma grave, pruebas de función respiratoria, biomarcadores, tratamiento con biológicos.

\section{INTRODUCCIÓN}

El asma afecta a 334,000,000 de personas en todo el mundo y aproximadamente $5-15 \%$ de los pacientes con asma se clasifican como enfermedad grave.' De acuerdo con la Guía de la Asociación Americana de Tórax y la Sociedad Europea Res-

\begin{abstract}
Severe asthma is a complex pathology with heterogeneous clinical features and underlying mechanisms (endotypes), being represented by a small group of patients, who present frequent exacerbations and obstruction of the airways, being refractory to standard treatment, requiring high doses of inhaled corticosteroids in combination with a second controller. Some even without presenting improvement despite this therapy. These are the severe uncontrolled asthmatics. New strategies have been achieved to control severe asthma, considering the components of the disease and the specific mechanisms of each phenotype; an important part has been the use of non-invasive biomarkers such as total serum IgE, the eosinophil count in peripheral blood and in sputum, and the determination of fractional exhaled nitric oxide (FeNO) for both, diagnosis, and follow-up. The development of biological therapies targeting specific inflammatory pathways in asthma have been a breakthrough in recent years, which are administered in selected patients to reduce exacerbations and improve disease control. In this manuscript we describe the perspectives of a group of experts in pulmonology and allergology on severe asthma.
\end{abstract}

Keywords: Severe asthma, respiratory function tests, biomarkers, biological treatment.

piratoria (ATS/ERS por sus siglas en inglés) publicada en 2020, un paciente con asma grave es aquel individuo que requiere un tratamiento con altas dosis de corticosteroides inhalados más un segundo controlador y/o corticosteroide sistémico para evitar que el paciente pierda el control de la enfermedad o que a pesar de esta terapia permanece sin control. ${ }^{2}$

\section{Correspondencia:}

Dra. Laura Gochicoa-Rangel

Instituto Nacional de Enfermedades Respiratorias Ismael Cosío Villegas, Ciudad de México, México.

Correo electrónico: laura.gochicoa@infire.mx

Recibido: 23-VII-2021; aceptado: 27-X-2021.

Citar como: Meneses-Tamayo E, Chavez-Torres W, Larenas-Linnemann D, Cano-Salas MC, Ortega-Martell JA, Gochicoa-Rangel L. Reporte breve: perspectivas médicas en asma grave. Neumol Cir Torax. 2021; 80 (4): 251-257. https://dx.doi.org/10.35366/103450 
En las últimas décadas se han realizado cambios importantes tanto en la evaluación como en el tratamiento del asma grave, ya que esta condición es responsable de una carga de enfermedad desproporcionalmente alta y de una mayor morbilidad. Se ha promovido la investigación y se han explorado más a fondo los fenotipos clínicos y los mecanismos celulares y moleculares (endotipos), identificando nuevos biomarcadores para su evaluación y seguimiento. ${ }^{3}$ Estos avances han permitido el desarrollo y uso de tratamientos biológicos, lo cual ha generado puntos de discusión en cuanto a su uso y cómo sumarlos al manejo clásico del asma de acuerdo con las comorbilidades de cada paciente, la exposición ambiental y la adherencia a la terapia para lograr el control de la enfermedad.,

Recientemente se realizó una sesión donde se expresaron diferentes enfoques acerca de la atención a pacientes con asma grave, centrándose en la evaluación clínica, bioquímica y funcional respiratoria así como en el uso de terapia biológica. El objetivo de este documento es dar a conocer la perspectiva del asma grave visto por un grupo de expertos en el área, conformado por dos alergólogos y dos neumólogos, los cuales respondieron a 13 preguntas que a continuación se resumen.

\section{Características clínicas de un paciente con asma grave}

Los pacientes con asma grave cursan con los síntomas cardinales de asma, de presentación intermitente e historial de exacerbaciones frecuentes que afectan su desempeño en actividades escolares y/o laborales, visitas frecuentes al servicio de urgencias, uso de corticosteroides sistémicos para lograr control de sintomatología y que en muchas ocasiones es incapacitante. ${ }^{3}$ Sin embargo, antes de catalogar al paciente como asma grave es necesario revisar el tratamiento y apego a los medicamentos e indicaciones, estableciendo si el asma es de difícil tratamiento debido a causas «extrínsecas» (falta de recursos económicos, tratamientos cortos, dosis no adecuadas o mala técnica al usar dispositivo de inhalación, exposición a irritantes, humo de cigarro, alérgenos o comorbilidades sin control); o «intrínseca» a la enfermedad. Una vez que se haya realizado el adecuado ajuste y escalamiento en el tratamiento, si se llega al paso 5 según el manejo integral del asma, lineamientos para México (MIA 2021) $)^{5}$ y de acuerdo con la iniciativa global para el asma (GINA por sus siglas en inglés) ${ }^{6}$ y el paciente no logra un control adecuado de la enfermedad, se puede establecer el diagnóstico de asma grave.

\section{Clasificación de asma grave}

De acuerdo con los criterios ERS, ${ }^{7}$ retomados por la Asociación Latinoamericana de Tórax (ALAT 2020) ${ }^{8}$ y el manejo integral del asma (MIA 2021), ${ }^{5}$ para abordar a un paciente con asma grave, éste debe clasificarse en asma grave controlada y asma grave no controlada; se utiliza al menos un criterio de los siguientes cuatro para definir asma grave no controlada:

a. Puntuación $<20$ en la prueba de control del asma (ACT) $\mathrm{o} \geq 1.5$ en el cuestionario de control del asma (ACQ).

b. Exacerbaciones frecuentes: dos o más esquemas de corticosteroides sistémicos ( $\geq 3$ días) en el año previo.

c. Exacerbaciones graves: al menos una hospitalización, admisión en la unidad de terapia intensiva (UTI) o ventilación mecánica invasiva en el último año.

d. Disminución en la función pulmonar: volumen espirado forzado en el primer segundo $\left(\mathrm{FEV}_{1}\right)<80 \%$ del predicho habiendo suspendido el broncodilatador previo a la espirometría basal.

Se puede clasificar de acuerdo con los mecanismos inflamatorios que desarrolla la patogenia:

a. Fenotipo inflamatorio tipo T2: asociado con mayor gravedad del asma, mayor frecuencia de exacerbaciones y función pulmonar disminuida; este fenotipo abarca dos formas, el asma alérgica y el asma eosinofílica no-alérgica.

b. Fenotipo no T2: no muestra evidencia de inflamación eosinofílica, más bien es neutrofílica o la inflamación eosinofílica es mínima. Se caracteriza porque el diagnóstico se realiza en la edad adulta, generalmente son individuos obesos, o con antecedente de tabaquismo o exposición a humo de segunda mano. ${ }^{3,9}$

\section{Evaluación integral del paciente con asma grave}

Es importante realizar una evaluación enfocada en la búsqueda de factores de riesgo, antecedentes o presencia de alergias (rinitis y conjuntivitis alérgicas, dermatitis atópica, alergia alimentaria) tanto en el paciente como en la familia, infecciones respiratorias recurrentes así como comorbilidades: obesidad, enfermedad por reflujo gastroesofágico, rinosinusitis, bronquiectasias, bronquiolitis, enfermedad cardiovascular, enfermedad intersticial pulmonar, enfermedad pulmonar obstructiva crónica. ${ }^{2,3}$

Complementar la evaluación con biometría hemática para ver recuento de eosinófilos en sangre, IgE total, IgE específica en suero o prueba cutánea en el paciente con sospecha de asma alérgica, espirometría prebroncodilatador y posbroncodilatador y si se cuenta con fracción exhalada de óxido nítrico (FeNO) realizarlo. ${ }^{8}$

\section{Evaluación de la función pulmonar en el paciente con asma grave}

La evaluación funcional respiratoria en el paciente con asma grave debe realizarse principalmente con pruebas 


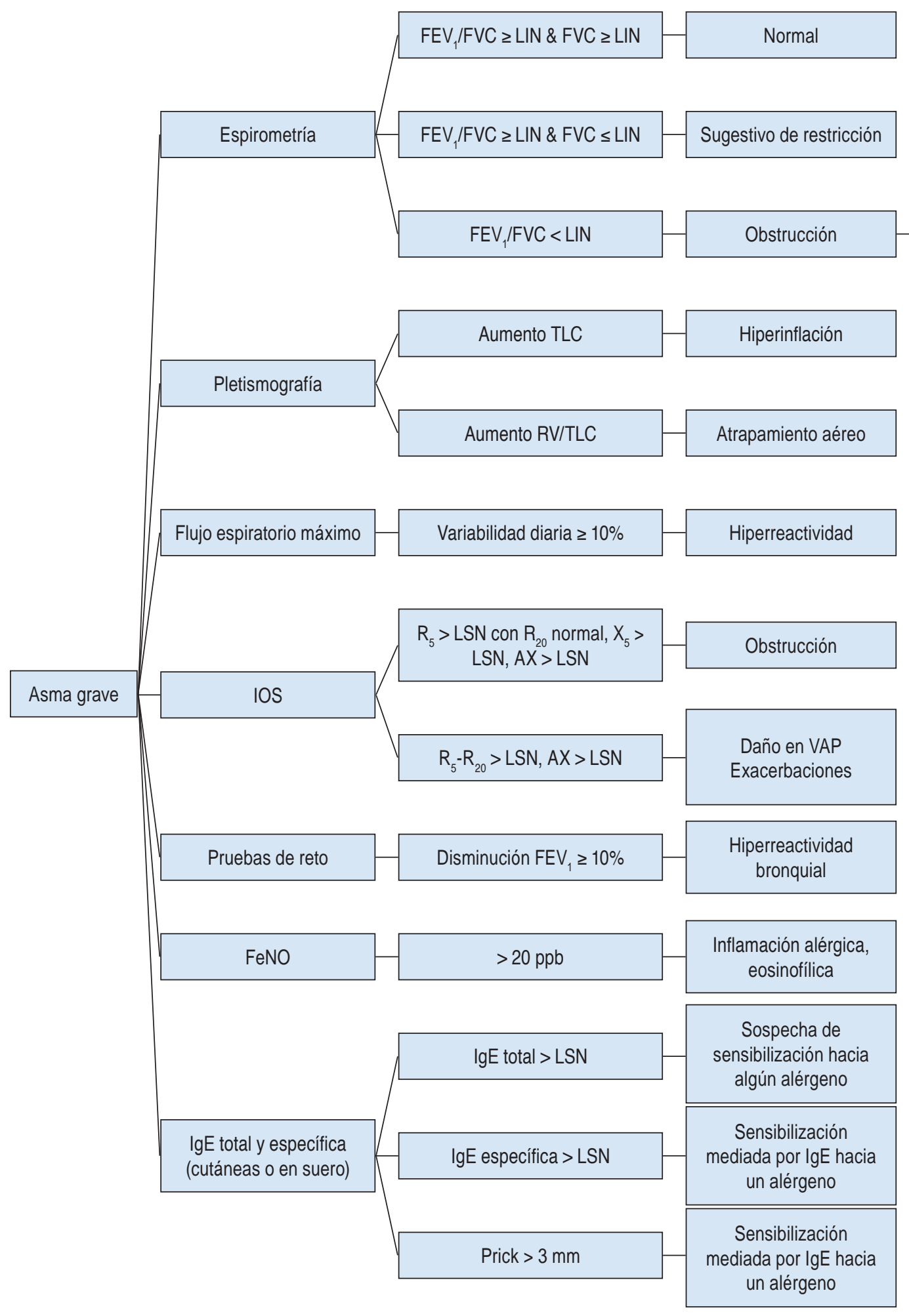

Figura 1: Pruebas de función respiratoria y biomarcadores inflamatorios en asma.

$\mathrm{FEV}_{1}=$ volumen espirado forzado en el primer segundo, FVC $=$ capacidad vital forzada, $\mathrm{LIN}=$ límite inferior de la normalidad, $\mathrm{BD}=$ broncodilatador, $\mathrm{TLC}=$ capacidad pulmonar total, $\mathrm{RV}=$ volumen residual, IOS = sistema de oscilometría de impulso, $\mathrm{R}_{5}=$ resistencias respiratorias a $5 \mathrm{Hertz}, \mathrm{LSN}=$ límite superior de la normalidad, $\mathrm{R}_{20}=$ resistencias respiratorias a $20 \mathrm{Hertz}, \mathrm{X} 5$ = reactancias respiratorias a $5 \mathrm{Hertz}, \mathrm{AX}=$ área de reactancia, VAP = vía aérea pequeña, FeNO = fracción exhalada de óxido nítrico, $\lg \mathrm{E}=$ inmunoglobulina $\mathrm{E}$. 
de mecánica respiratoria que permitan conocer el estado funcional del individuo como espirometría, pletismografía y oscilometría y mediante biomarcadores de inflamación eosinofílica como la fracción exhalada de óxido nítrico (FeNO). En la Figura 1 se observan las principales pruebas sugeridas.

El estudio inicial es la espirometría, la cual mostrará obstrucción que con el tiempo y el mal control de la enfermedad puede ser fija o sólo parcialmente reversible. ${ }^{10}$ Los pacientes con frecuencia cursan con atrapamiento aéreo y capacidad vital forzada (FVC) disminuida. Existe un aumento en el volumen residual (RV) y en la capacidad residual funcional (FRC), por lo que el índice RV/TLC (capacidad pulmonar total) se encuentra elevado. En estos pacientes en ocasiones es necesario hacer pletismografía que adicionalmente mostrará aumento en las resistencias de la vía aérea. ${ }^{11}$ El seguimiento mediante flujometría mostrará variabilidad diaria de más de $10 \%$. Una prueba que se realiza de manera cada vez más frecuente es la oscilometría, en estos pacientes se esperan resistencias a bajas frecuencias (R5) muy elevadas, con un área de reactancia $(A X)$ también incrementada. Generalmente estos sujetos cursan con pruebas de reto bronquial positivas que traducen el mal control de la enfermedad. Otro componente importante es que, a pesar de la utilización de esteroides a dosis altas, la fracción exhalada de óxido nítrico puede encontrarse $>25 \mathrm{ppb}$ en adultos o > 20 ppb en niños, en inflamación eosinofílica (T2), con deterioro en la función pulmonar y mayor tasa de exacerbaciones. ${ }^{12,13}$

Existen otros estudios como la difusión pulmonar de monóxido de carbono (DLCO) que puede ayudar a descartar otras probabilidades diagnósticas, aunque habitualmente en el asma puede estar aumentada. Algunas personas con asma grave cursan con heterogeneidad de la ventilación, por lo que la medición del índice de aclaramiento pulmonar ( $\mathrm{LCl}$ por sus siglas en inglés) podría ser de utilidad, el cual se encontraría aumentado. ${ }^{11,14}$

\section{Evaluación funcional respiratoria en pacientes con asma grave durante la pandemia de la enfermedad por coronavirus en 2019 (COVID-19)}

Durante el inicio de la pandemia por el SARS-CoV-2, los laboratorios de función pulmonar se cerraron y se realizaban sólo estudios que fueran estrictamente necesarios para la toma de alguna decisión clínica; sin embargo, pese a la indicación médica y a la adaptación de protocolos de bioseguridad dentro de los laboratorios no se hacían pruebas para seguimiento de esta enfermedad. Debido al riesgo de contagio se suspendieron las espirometrías en consultorio y se optó por hacer más énfasis en los cuestionarios de control de síntomas, la oximetría de pulso domiciliaria y en consultorio así como la instrucción y realización de flujometría en el domicilio del paciente con registro diario para evaluar la variabilidad del flujo espiratorio pico. Actualmente ya se efectúan todos los estudios de función respiratoria y existen diferentes consensos, los cuales coinciden en la importancia de la ventilación en el área donde se realizan los estudios, y el uso del equipo de protección personal por parte del personal de salud. ${ }^{15}$

\section{Biomarcadores en asma grave y su utilidad}

Los biomarcadores se utilizan principalmente para identificar individuos con inflamación de las vías respiratorias tipo T2. Dentro de éstos, se pueden solicitar:

a. Eosinófilos en esputo: es el más característico de inflamación T2, y se ha propuesto como un factor predictor de respuesta en el uso de terapia con biológicos como anti-IL5, anti-IL5R y anti-IL4/13R, ya que han mostrado un gran beneficio en estos pacientes; disminuyen las exacerbaciones y mejoran la calidad de vida. ${ }^{8}$

b. FeNO: fácilmente medible, siendo el resultado del aumento en la expresión del óxido nítrico sintasa, inducida por la IL-13. En adultos con FeNO > 25 ppb y niños con $>20$ ppb es indicativo de inflamación eosinofílica en pacientes sintomáticos y en adultos con $>50 \mathrm{ppb}$ y niños con > 35 ppb es indicativo de inflamación eosinofílica grave. ${ }^{12,13}$ Sin embargo, por el uso de Corticoesteroides inhalados (CSI) en dosis altas se puede encontrar normal en el asma grave controlada.

c. Recuento diferencial de eosinófilos en sangre (EOS): el punto de corte de eosinófilos en sangre periférica es de $300 / \mu \mathrm{L}$. Se debe tener en cuenta que el conteo de eosinófilos puede estar disminuido por el uso de corticosteroides sistémicos e inhalados. Su determinación resulta de mucha utilidad para evaluar la respuesta a la administración de anticuerpos monoclonales antiIL5 y anti-IL5R, ya que se obtiene mejor respuesta con cifras elevadas de eosinófilos en sangre. Se han establecido distintos puntos de corte de acuerdo con cada anticuerpo monoclonal, siendo $\geq 150 / \mu \mathrm{L}$ para mepolizumab, $\geq 300 / \mu \mathrm{L}$ para benralizumab y $\geq 400 /$ $\mu \mathrm{L}$ para reslizumab. ${ }^{3,6,8}$

d. IgE: es un mal predictor de la eosinofilia de las vías respiratorias, por lo que la ATS/ERS recomienda utilizar IgE sérica entre 30-700 UI/mL (aunque algunos autores sugieren un máximo de 1,200 UI/mL), en conjunto con los valores de eosinófilos en sangre periférica $\geq 260 / \mu \mathrm{L}$ y $\mathrm{FeNO} \geq 19.5$ ppb como guía para iniciar tratamiento con omalizumab, ya que la IgE sérica no se asocia con respuesta a otros tratamientos. ${ }^{2,7}$ 


\section{Inicio de tratamiento biológico}

Se debe valorar la adherencia al tratamiento previamente indicado así como el tratamiento de las comorbilidades diagnosticadas, y si las intervenciones realizadas han llevado al control del paciente. En caso contrario, conocer el impacto de la sintomatología en la calidad de vida al desempeñar sus actividades académicas, laborales, familiares; y verificar el tiempo de tratamiento con dosis elevadas de corticosteroides tanto inhalados como sistémicos, es importante conocer si han sido administrados por más de tres a seis meses. Si a pesar de todo lo anterior el paciente continúa presentando exacerbaciones, se deberá valorar la prescripción de un anticuerpo monoclonal.

\section{Adulto: asma grave}
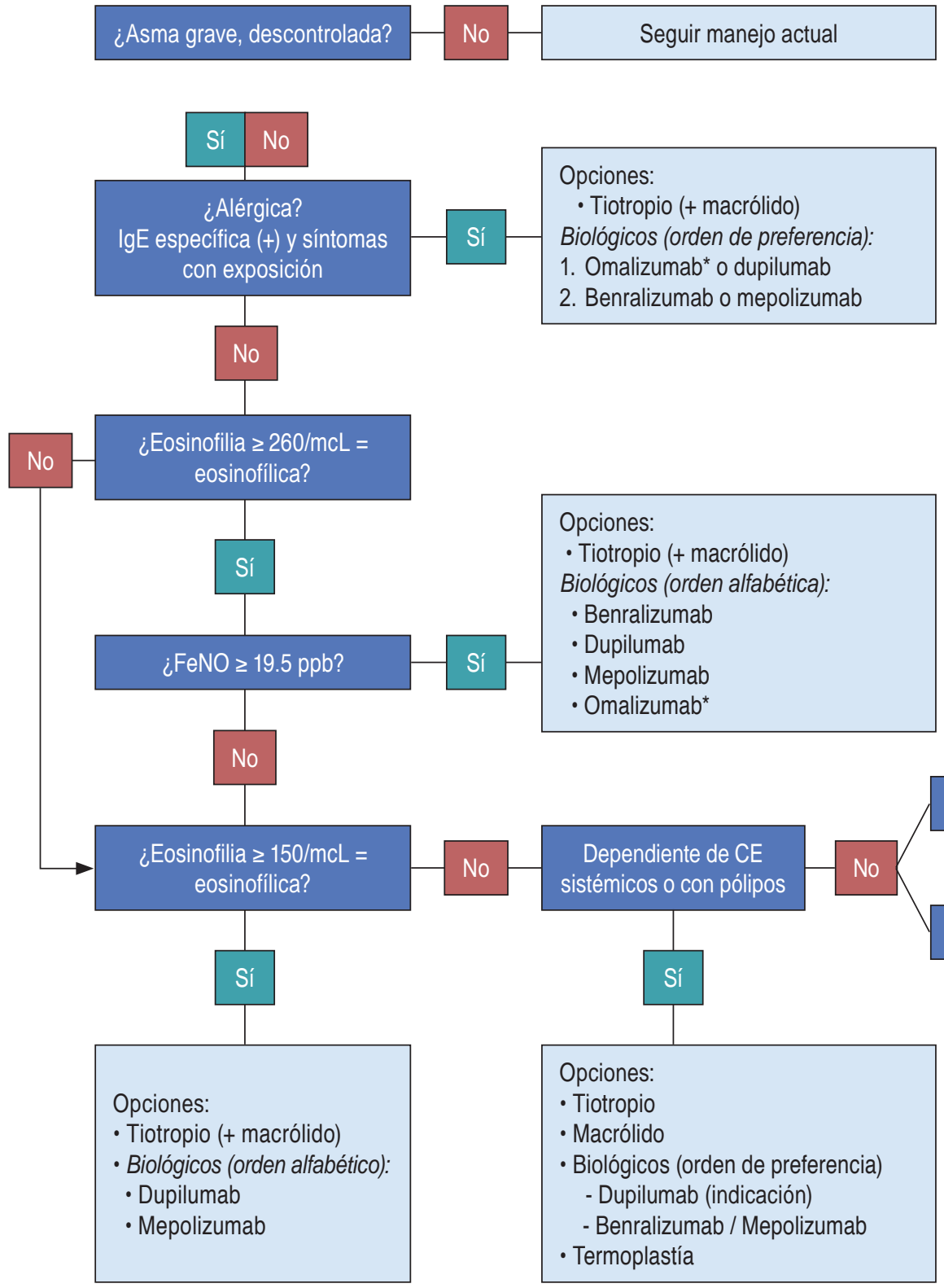

Sí Biológicos (orden de preferencia):

1. Omalizumab* o dupilumab

2. Benralizumab o mepolizumab $\geq 6$ años: omalizumab (anti-lgE)

$\geq 12$ años: mepolizumab, reslizumab

(anti-IL5); dupilumab (anti-IL4/IL13)

$\geq 18$ años: benralizumab

(antirreceptor IL5)

Figura 2: Flujograma de evaluación y tratamiento del asma grave. 


\section{Tratamiento con anticuerpos monoclonales de acuerdo con los biomarcadores}

Para la prescripción del tratamiento con biológicos se toman en cuenta las características clínicas de cada paciente y los resultados de los biomarcadores (IgE, EOS y FeNO), para eso nos podemos guiar por el algoritmo sugerido por la guía de MIA, ${ }^{5}$ u otras sociedades respiratorias $2,6,16,17$ y de esta manera optimizar el uso de corticosteroides sistémicos y lograr la disminución de los mismos (Figura 2).

\section{Seguimiento del paciente en tratamiento con biológico}

Se sugiere evaluar a los pacientes después de tres a seis meses de terapia, teniendo en cuenta la disminución en la tasa de exacerbaciones, la mejoría subjetiva en el control de los síntomas, la función pulmonar, los efectos secundarios, la reducción en la dosis de corticosteroides, la calidad de vida y la satisfacción del paciente de acuerdo con la respuesta al tratamiento. Además de lo anterior, se podría considerar la toma de biomarcadores séricos asociados con la inflamación T2 para evaluar la respuesta al tratamiento de acuerdo con el medicamento biológico empleado. ${ }^{6,9}$

\section{Seguimiento del paciente con asma} grave mediante biomarcadores

En general, se debe revisar la respuesta del paciente a la terapia biológica después de tres a cuatro meses, los puntos más importantes a evaluar son: ${ }^{2-4,6,8}$

a. Control de los síntomas: a través de los cuestionarios $\mathrm{ACT}, \mathrm{ACQ}$.

b. Número de exacerbaciones y gravedad.

c. Necesidad de usar corticosteroides orales para exacerbaciones o dosis de mantenimiento con presencia de efectos secundarios derivados del uso crónico de corticosteroides sistémicos.

d. Presencia de disminución en la función pulmonar comparando con estudios previos.

e. Control adecuado de las comorbilidades.

f. Recuento de EOS y FeNO, aunque es muy importante tomar en cuenta que estas mediciones se pueden ver afectadas por el uso de corticosteroides y del medicamento biológico utilizado; por ello en general no deben guiar el tratamiento.

\section{Disminución de la dosis de corticosteroides orales}

Para disminuir la dosis de costicosteroides orales se debe tomar en cuenta la prevención de la reactivación de la enfermedad y el déficit de cortisol por supresión del eje hipotálamo-hipófisis-suprarrenal (HHS), programando el retiro de los corticosteroides orales en función de los niveles de cortisol, ${ }^{18}$ las pruebas de función respiratoria y la toma de biomarcadores para valorar la respuesta al tratamiento biológico.

Se pueden suspender de manera inmediata los tratamientos crónicos con menos de $5 \mathrm{mg} /$ día de prednisolona o su dosis equivalente y los que llevan menos de tres semanas, independientemente de la dosis, vigilando de cerca datos de insuficiencia suprarrenal y/o la presencia de una exacerbación. ${ }^{19}$

En tratamientos prolongados con más de $5 \mathrm{mg}$ /día de prednisolona o su dosis equivalente se deberá disminuir de manera progresiva la dosis diaria del corticosteroide: ${ }^{19}$

a. $10 \mathrm{mg} /$ día cada una a dos semanas, cuando la dosis inicial de prednisolona (o equivalente) es $>40 \mathrm{mg} / \mathrm{día}$.

b. $5 \mathrm{mg} /$ día cada una a dos semanas, cuando la dosis de prednisolona se encuentra entre $20-40 \mathrm{mg} /$ día.

c. $2.5 \mathrm{mg} /$ día cada dos a tres semanas, cuando la dosis de prednisolona está entre 10-19 mg/día.

d. $1 \mathrm{mg} /$ día cada dos a cuatro semanas, cuando la dosis de prednisolona sea menor de $10 \mathrm{mg} /$ día.

Siempre considerar las comorbilidades con las que curse el paciente, ya que en algunas enfermedades, por ejemplo las vasculitis, no se podrá retirar de manera completa el corticosteroide.

\section{Retiro de biológico}

Cuando se tiene un paciente con adecuada respuesta al tratamiento con biológico, se considera el retiro del mismo no antes de 12 meses de tratamiento como mínimo, siempre y cuando el asma del paciente ha cursado con un buen control utilizando una dosis media de corticosteroide inhalado; y de existir asma alérgica, no tener exposición al agente desencadenante previamente identificado; ${ }^{2,6,9}$ sin embargo, el tiempo de uso dependerá de la evolución clínica-funcional del paciente y por el momento no es posible generalizar la duración del tratamiento.

\section{CONCLUSIÓN}

Los pacientes con asma grave cursan con exacerbaciones frecuentes y obstrucción de la vía aérea, muchos de ellos requieren altas dosis de esteroide inhalado en combinación con otros medicamentos. La terapia con biológicos es una nueva alternativa de tratamiento que requiere un seguimiento estrecho de la función respiratoria y de biomarcadores inflamatorios.

\section{Agradecimientos}

Al Dr. Armando Partida Gaytán por la organización de esta actividad, la cual fue auspiciada por la compañía GSK; sin embargo, la opinión de los expertos y el presente documento son independientes de dicha compañía. 


\section{REFERENCIAS}

1. Zein JG, Dweik RA, Comhair SA, Bleecker ER, Moore WC, Peters $\mathrm{SP}$, et al. Asthma is more severe in older adults. PLoS One. 2015;10:e0133490.

2. Holguin F, Cardet JC, Chung KF, Diver S, Ferreira DS, Fitzpatrick A, et al. Management of severe asthma: a European Respiratory Society/ American Thoracic Society guideline. Eur Respir J. 2020;55:1900588.

3. Cote A, Godbout K, Boulet LP. The management of severe asthma in 2020. Biochem Pharmacol. 2020;179:114112.

4. Pike KC, Levy ML, Moreiras J, Fleming L. Managing problematic severe asthma: beyond the guidelines. Arch Dis Child. 2018;103:392-397.

5. Larenas-Linnemann D, Salas-Hernández J, Del-Rio-Navarro B, LunaPech JA, Navarrete-Rodríguez EM, Gochicoa-Rangel L, et al. MIA 2021. MIA 2021, Comprehensive Asthma Management, Guidelines for Mexico. Rev Alerg Mex. 2021;68 Suppl 1:s1-s122.

6. Global Initiative for Asthma. Difficult-to-treat \& severe asthma in adolescent and adult patients. Diagnosis and management. 2019 [Access: 14 July 2021]. Available in: https://ginasthma.org/wp-content/ uploads/2019/04/GINA-Severe-asthma-Pocket-Guide-v2.0-wms-1.pdf.

7. William BW. Severe asthma: definition and impact. European Respiratory Society. 2019;1-15.

8. Asociación Latinoamericana de Tórax ALAT. Guía de Práctica Clínica Latinoamericana de Asma Grave. Basada en evidencia. 2020. Disponible en: https://alatorax.org/es/guias/guia-de-practica-clinicalatinoamericana-en-asma-grave-alat-2020-1

9. Reihman AE, Holguin F, Sharma S. Management of severe asthma beyond the guidelines. Curr Allergy Asthma Rep. 2020;20:47.

10. Global Initiative for Asthma. Global strategy for asthma management and prevention. 20212021 [Cited 2021 Julio 14]. Available in: https:// ginasthma.org/wp-content/uploads/2021/05/GINA-Main-Report2021-V2-WMS.pdf
11. O'Toole J, Mikulic L, Kaminsky DA. Epidemiology and pulmonary physiology of severe asthma. Immunol Allergy Clin North Am. 2016;36:425-438.

12. Menzies-Gow A, Mansur AH, Brightling $\mathrm{CE}$. Clinical utility of fractional exhaled nitric oxide in severe asthma management. Eur Respir J. 2020;55(3):1901633.

13. Larenas-Linnemann D, Gochicoa-Rangel L, Macías-Weinmann A, SotoRamos M, Luna-Pech JA, Elizondo-Ríos A, et al. Consenso mexicano en relación con la fracción exhalada de óxido nítrico (FeNO) en asma 2020. Rev Alerg Mex. 2020;67:s1-s25.

14. King GG, James A, Harkness L, Wark PAB. Pathophysiology of severe asthma: we've only just started. Respirology. 2018;23:262-271.

15. Gochicoa-Rangel L, Torre-Bouscoulet L, Salles-Rojas A, GuzmánValderrábano C, Silva-Cerón M, Benítez-Pérez R, et al. Functional respiratory evaluation in the COVID-19 era: the role of pulmonary function test laboratories. Rev Invest Clin. 2020;73:10.24875/ RIC.20000250.

16. British Thoracic Society. British guideline on the management of asthma. 2019. Available in: https://www.brit-thoracic.org.uk/qualityimprovement/guidelines/asthma/

17. Sociedad Española de Neumología y Cirugía Torácica. Guía Española Para el Manejo del Asma. 2020. Available in: www.gemasma.com

18. Williams DM. Clinical pharmacology of corticosteroids. Respiratory Care. 2018;63:655-670.

19. Guerrero PF, Marengo AP, Villabona Artero C. The unresolved riddle of glucocorticoid withdrawal. J Endocrinol Invest. 2017;40(11):11751181.

Conflicto de intereses: Los autores declaran no tener conflicto de intereses. 